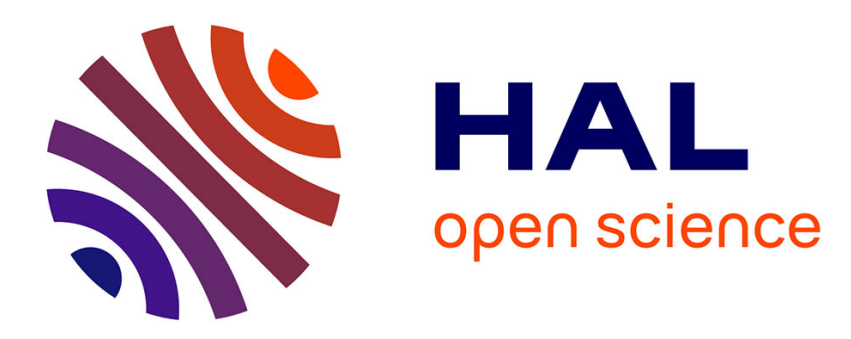

\title{
A Relaxation Result for State-Constrained Delay Differential Inclusions
}

Hélène Frankowska, Ihab Haidar

\section{To cite this version:}

Hélène Frankowska, Ihab Haidar. A Relaxation Result for State-Constrained Delay Differential Inclusions. IEEE Transactions on Automatic Control, 2018, 63 (11), pp.3751-3760. 10.1109/TAC.2018.2794398 . hal-02126119

\section{HAL Id: hal-02126119 \\ https://hal.science/hal-02126119}

Submitted on 10 May 2019

HAL is a multi-disciplinary open access archive for the deposit and dissemination of scientific research documents, whether they are published or not. The documents may come from teaching and research institutions in France or abroad, or from public or private research centers.
L'archive ouverte pluridisciplinaire HAL, est destinée au dépôt et à la diffusion de documents scientifiques de niveau recherche, publiés ou non, émanant des établissements d'enseignement et de recherche français ou étrangers, des laboratoires publics ou privés. 


\title{
A relaxation result for state constrained delay differential inclusions
}

\author{
HÉLÈNE FRANKOWSKA ${ }^{1}$ AND IHAB HAIDAR ${ }^{2}$ \\ ${ }^{1}$ CNRS, IMJ-PRG, Sorbonne Universités, UPMC Université Paris 6, \\ Case 247, 4 Place Jussieu, 75252 Paris, France \\ ${ }^{2}$ Sorbonne Universités, IMJ-PRG, UPMC Université Paris 6, \\ 4 Place Jussieu, 75252 Paris, France
}

\begin{abstract}
In this paper, we consider a delay differential inclusion $\dot{x}(t) \in F\left(t, x_{t}\right)$, where $x_{t}$ denotes the history function of $x(t)$ along an interval of time. We extend the celebrated Filippov's theorem to this case. Then, we further generalize this theorem to the case when the state variable $x$ is constrained to the closure of an open subset $K \subset \mathbb{R}^{n}$. Under a new "inward pointing condition", we give a relaxation result stating that the set of trajectories lying in the interior of the state constraint is dense in the set of constrained trajectories of the convexified inclusion $\dot{x}(t) \in \operatorname{co} F\left(t, x_{t}\right)$.
\end{abstract}

Index Terms-Delay differential inclusions, relaxation, state constraints, inward pointing conditions.

\section{INTRODUCTION}

Mathematical models arising in population dynamics or engineering sciences often involve control systems with delays (see, e.g., [6], [24]). Systems with delays, express that at each instant the velocity of the state depends upon the history of its evolution up to this instant [23]. Such control systems can be described as follows:

$$
\left\{\begin{array}{rlrl}
\dot{x}(t) & =f\left(t, x_{t}, u(t)\right), & & \text { a.e. } t \in\left[t_{0}, T\right], \\
u(t) & \in U \subset \mathbb{R}^{q}, & & \text { a.e. } t \in\left[t_{0}, T\right], \\
x_{t_{0}} & =\varphi, &
\end{array}\right.
$$

where $x(t) \in \mathbb{R}^{n}$, represents the state at time $t$, $x_{t}:[-\tau, 0] \rightarrow \mathbb{R}^{n}$ is the standard notation for the history function defined by $x_{t}(\theta)=x(t+\theta)$, for $\tau>0$ and $-\tau \leq \theta \leq 0, u(\cdot)$ is a Lebesgue measurable function, $f$ is a mapping from $[0, T] \times \mathcal{C}\left([-\tau, 0], \mathbb{R}^{n}\right) \times U$ into $\mathbb{R}^{n}, 0 \leq t_{0} \leq T$, and $\varphi$ is the initial condition taken in $\mathcal{C}\left([-\tau, 0], \mathbb{R}^{n}\right)$. In the above, $\mathcal{C}\left([-\tau, 0], \mathbb{R}^{n}\right)$ denotes the Banach space of continuous functions from $[-\tau, 0]$ into $\mathbb{R}^{n}$, with the usual norm.

This work was partially funded by the DeMagma project of Programme Convergence from Idex Super at Sorbonnes Universités.
When the trajectories of (1) are subject to the state constraint

$$
x(t) \in K \quad \forall t \in\left[t_{0}, T\right],
$$

where $K$ is a closed subset of $\mathbb{R}^{n}$, the viability theory [2] provides adequate mathematical tools to study the existence of feasible (or viable) solutions of such systems. Thanks to this theory, a necessary and sufficient condition (linking the dynamics of system (1) to the geometry of the constraint set $K$ ) for the existence of feasible solutions is known. Under some regularity assumptions on $f$, this condition was first given in [21]:

$$
\begin{gathered}
\forall t \in[0, T], \forall \psi \in \mathcal{C}\left([-\tau, 0], \mathbb{R}^{n}\right) \text { such that } \psi(0) \in K, \\
f(t, \psi, U) \cap T_{K}(\psi(0)) \neq \emptyset,
\end{gathered}
$$

where $T_{K}(\psi(0))$ is the contingent cone to $K$ at $\psi(0)$. In the framework of this theory, convexity conditions are imposed on the set-valued map $F(t, \psi):=f(t, \psi, U)$, i.e. for every $t \in[0, T]$ and every $\psi \in \mathcal{C}\left([-\tau, 0], \mathbb{R}^{n}\right)$, $F(t, \psi)$ is a convex subset of $\mathbb{R}^{n}$. This convexity hypothesis may fail in some mathematical models and may be even difficult to verify.

In the case of delay-free control systems, a vast literature (see, e.g., [8], [9], [10], [13], [15], [19], [20]) allows to relax this convexity hypothesis, by assuming, as a counterpart, stronger tangential conditions and stronger regularity of $F$. These conditions rely on the possibility of directing a velocity into the interior of the constraint $K$ whenever approaching the boundary of $K$. Known as inward pointing conditions, they allow to approximate relaxed feasible trajectories by feasible trajectories and provide estimates on the distance of a given trajectory of unconstrained control system from the set of feasible trajectories, see for instance [8], [9], [15], [17], [19]. In the literature, these estimates have been referred to as neighboring feasible trajectory (NFT) estimates. In the case when $F$ is Lebesgue measurable with respect 
to the time and Lipschitz with respect to the state, NFT estimates result from the following inward pointing condition (see [16], [17]):

$$
\left\{\begin{array}{c}
\forall t \in[0, T], \forall x \in \partial K, \forall v \in F(t, x) \\
\text { such that } \max _{n \in N_{K}^{1}(x)}\langle n, v\rangle \geq 0, \\
\exists w \in \operatorname{Liminf}_{(s, y) \rightarrow(t, x)} \operatorname{co} F(s, y) \\
\text { satisfying } \max _{n \in N_{K}^{1}(x)}\langle n, w-v\rangle<0,
\end{array}\right.
$$

where $\operatorname{co} F(s, y)$ is the convex hull of $F(s, y)$, Liminf denotes the Kuratowski lower set limit (see [3]), $N_{K}^{1}(x):=N_{K}(x) \cap S^{n-1}, S^{n-1}$ is the unit sphere and $N_{K}(x)$ denotes the Clarke normal cone to $K$ at $x$ (see [11]). The above condition takes sometimes a simpler form depending on the regularity assumptions on $F$ and the smoothness of the boundary $\partial K$ (see, e.g., [8], [9], [10], [17], [20]).

When the viability condition fails to be fulfilled on the boundary of $K$, the largest subset of initial conditions starting from which at least one viable solution exists (called viability kernel) is considered. In the case of delay-free control systems, viability algorithms providing constructive methods for the computation of the viability kernel, have been developed (see, e.g., [18], [28]). Thanks to these algorithms, efficient numerical methods have been established (see, e.g., [27]) and used in order to exhibit approximating viability kernels for numerous examples coming from different fields (see, e.g., [4], [7], [29], [25], [22], [26]). We would like to underline that these algorithms are developed for setvalued maps with convex values. Two steps are needed to extended these numerical methods to delay differential inclusions: adapt the viability algorithms to this case and obtain relaxation theorems under state constraints. This latter point is the purpose of this paper.

To our knowledge, NFT estimates for delay differential inclusions are not yet obtained in the literature. Here, we propose to extend such results to this case. Inspired by the viability condition given by (3), we propose to adapt the inward pointing condition (4) to delay differential inclusions.

Let $\lambda>0$. Define the set

$$
\begin{gathered}
\mathcal{K}_{\lambda}:=\left\{\psi \in \mathcal{C}\left([-\tau, 0], \mathbb{R}^{n}\right): \psi \text { is } \lambda\right. \text {-Lipschitz } \\
\text { and } \psi(0) \in \partial K\}
\end{gathered}
$$

and consider the following relaxed inward pointing condition:

$$
\left(I P_{r e l}^{\lambda}\right)\left\{\begin{array}{l}
\forall t \in[0, T], \forall \psi \in \mathcal{K}_{\lambda}, \forall v \in F(t, \psi) \\
\text { such that } \max _{n \in N_{K}^{1}(\psi(0))}\langle n, v\rangle \geq 0, \\
\exists w \in \operatorname{Liminf}_{(s, \phi) \rightarrow(t, \psi)} \operatorname{co} F(s, \phi) \\
\text { satisfying } \max _{n \in N_{K}^{1}(\psi(0))}\langle n, w-v\rangle<0 .
\end{array}\right.
$$

Assuming $\left(I P_{r e l}^{\lambda}\right)$, we give a relaxation result stating that the set of feasible trajectories is dense in the set of relaxed feasible ones. This is proved by using several preliminary results. The first one is an extension of the Filippov theorem, given by [12], to delay differential inclusions, which is an essential step to construct feasible trajectories. Then, we provide NFT estimates on the distance of a given trajectory from the set of feasible trajectories.

The paper is organized as follows. Section II presents the list of notations, definitions and assumptions in use. In Section III we state our main results. The proofs and useful technical tools are postponed to Section V. In Section IV we discuss an example, where the model corresponds to the problem considered in the present work.

\section{PRELIMINARIES}

In this section we list the notations and the main assumptions in use.

\section{A. Notations and definitions}

Consider the Euclidean space $\left(\mathbb{R}^{n},\|\cdot\|\right)$, where $n$ is a positive integer. We denote by $\langle\cdot, \cdot\rangle$ the inner product, by $B(x, r)$ the closed ball of center $x \in \mathbb{R}^{n}$ and radius $r>0$ and by $B$ the closed unit ball in $\mathbb{R}^{n}$ centered at 0 . Let co $A$ stands for the convex hull of a subset $A \subset \mathbb{R}^{n}$. For every pair $(a, b) \in \mathbb{R}^{2}$, set $a \vee b=\max \{a, b\}$ and $a \wedge b=\min \{a, b\}$.

Given $I \subset \mathbb{R},\left(\mathcal{C}\left(I, \mathbb{R}^{n}\right),\|\cdot\|_{C}\right)$ denotes the Banach space of continuous functions from $I$ into $\mathbb{R}^{n}$, where $\|\cdot\|_{C}$ is the norm of uniform convergence. Given $\tau>0, B_{C}(\varphi, r)$ denotes the closed ball of center $\varphi \in \mathcal{C}\left([-\tau, 0], \mathbb{R}^{n}\right)$ and radius $r>0$ and $B_{C}$ is the closed unit ball in $\mathcal{C}\left([-\tau, 0], \mathbb{R}^{n}\right)$ centered at 0 . Given $t \in \mathbb{R}$, we denote by $B((t, \varphi), r)$ the closed ball $B(t, r) \times B_{C}(\varphi, r)$.

We denote by $\mu$ the Lebesgue measure on the real line, and by $\mathcal{L}^{1}\left(I, \mathbb{R}^{n}\right)$ the space of Lebesgue integrable functions from $I$ to $\mathbb{R}^{n}$. 
Let $K$ be a nonempty closed subset of $\mathbb{R}^{n}$, Int $K$ be its interior and $\partial K$ its boundary, $\tilde{d}_{K}$ is the oriented distance from $x \in \mathbb{R}^{n}$ to $K$ defined by

$$
\tilde{d}_{K}(x)= \begin{cases}d_{K}(x) & \text { if } x \notin K \\ -d_{\mathbb{R}^{n} \backslash K}(x) & \text { otherwise, }\end{cases}
$$

where $d_{K}(x)=\inf _{y \in K}\|x-y\|$.

We will use the following notion of solution:

Definition 1: Let $0 \leq t_{0} \leq T, \tau>0$ and $\varphi \in$ $\mathcal{C}\left([-\tau, 0], \mathbb{R}^{n}\right)$. A function $x \in \mathcal{C}\left(\left[t_{0}-\tau, T\right], \mathbb{R}^{n}\right)$ is called an F-trajectory, if $x(\cdot)$ is absolutely continuous on $\left[t_{0}, T\right]$ and

$$
\begin{aligned}
\dot{x}(t) & \in F\left(t, x_{t}\right) \quad \text { a.e. } t \in\left[t_{0}, T\right], \\
x_{t_{0}} & =\varphi .
\end{aligned}
$$

An $F$-trajectory which verifies the state constraint (2) is called feasible $F$-trajectory. A trajectory associated to the relaxed differential inclusion

$$
\left\{\begin{aligned}
\dot{x}(t) & \in \operatorname{co} F\left(t, x_{t}\right), \quad \text { a.e. } t \in\left[t_{0}, T\right], \\
x_{t_{0}} & =\varphi
\end{aligned}\right.
$$

is called relaxed $F$-trajectory, and relaxed feasible $F$ trajectory if in addition (2) holds true.

\section{B. Assumptions}

Let $0 \leq t_{0} \leq T, \tau>0$ and $F:\left[t_{0}, T\right] \times$ $\mathcal{C}\left([-\tau, 0], \mathbb{R}^{n}\right) \rightsquigarrow \mathbb{R}^{n}$ be a set-valued map with nonempty closed images. In our main theorems, we will assume the following regularity conditions on $F$ :

(H1) for every $\psi \in \mathcal{C}\left([-\tau, 0], \mathbb{R}^{n}\right)$

$$
\text { the set-valued map } F(\cdot, \psi) \text { is measurable; }
$$

(H2) the set-valued map $F(t, \cdot)$ is locally Lipschitz in the following sense: $\forall R>0$, $\exists \zeta_{R}(\cdot) \in \mathcal{L}^{1}\left(\left[t_{0}, T\right], \mathbb{R}^{+}\right)$such that, for a.e. $t \in\left[t_{0}, T\right]$ and any $\varphi, \psi \in R B_{C}$

$$
F(t, \varphi) \subset F(t, \psi)+\zeta_{R}(t)\|\varphi-\psi\|_{C} B ;
$$

(H3) the set-valued map $F$ has a sublinear growth, i.e. there exists $\sigma>0$ such that, for a.e. $t \in\left[t_{0}, T\right]$ and any $\psi \in \mathcal{C}\left([-\tau, 0], \mathbb{R}^{n}\right)$

$$
F(t, \psi) \subset \sigma\left(1+\|\psi\|_{C}\right) B
$$

(H4) for a given $\lambda>0$, the set-valued map $F$ is upper semicontinuous on $\left[t_{0}, T\right] \times \mathcal{K}_{\lambda}$, i.e. for all $t \in\left[t_{0}, T\right]$ and all $\varphi \in \mathcal{K}_{\lambda}$, we have $F(t, \varphi) \neq \emptyset$ and for every $\varepsilon>0$ there exists $\delta>0$ such that

$$
F(s, \psi) \subset F(t, \varphi)+\varepsilon B \quad \forall(s, \psi) \in B((t, \varphi), \delta) .
$$

\section{MAIN RESUlTS}

\section{A. Filippov's Theorem}

The following theorem extends the celebrated Filippov's theorem, given by [12], to differential inclusions of type (6).

Theorem 1: Let $\beta>0$ and $\delta_{0} \geq 0$ and assume (H1), (H2). Let $y \in \mathcal{C}\left(\left[t_{0}-\tau, T\right], \mathbb{R}^{n}\right)$ be such that $y(\cdot)$ is absolutely continuous on $\left[t_{0}, T\right]$. Set $R=\max _{t \in\left[t_{0}-\tau, T\right]}\|y(t)\|$,

$$
\begin{aligned}
& \gamma_{1}(t)=d_{F\left(t, y_{t}\right)}(\dot{y}(t)), \\
& \gamma_{2}(t)=\exp \left\{\int_{t_{0}}^{t} \zeta_{R+\beta}(s) d s\right\}, \\
& \gamma_{3}(t)=\gamma_{2}(t)\left(\delta_{0}+\int_{t_{0}}^{t} \gamma_{1}(s) d s\right) .
\end{aligned}
$$

If $\gamma_{3}(T)<\beta$, then for all $\varphi \in \mathcal{C}\left([-\tau, 0], \mathbb{R}^{n}\right)$ with $\left\|\varphi-y_{t_{0}}\right\|_{C} \leq \delta_{0}$, there exists $x \in \mathcal{C}\left(\left[t_{0}-\tau, T\right], \mathbb{R}^{n}\right)$ such that $x(\cdot)$ is an $F$-trajectory and for all $t \in\left[t_{0}, T\right]$

$$
\left\|x_{t}-y_{t}\right\|_{C} \leq \gamma_{3}(t)
$$

and for almost every $t \in\left[t_{0}, T\right]$,

$$
\|\dot{x}(t)-\dot{y}(t)\| \leq \zeta_{R+\beta}(t) \gamma_{3}(t)+\gamma_{1}(t) .
$$

The following theorem establishes the possibility of approximating any relaxed $F$-trajectory by an $F$-trajectory starting from the same initial condition.

Theorem 2: Let $y(\cdot)$ be a relaxed $F$-trajectory. Assume (H1), (H2) and (H3). Then for every $\delta>0$ there exists an F-trajectory $x(\cdot)$ satisfying $x_{t_{0}}=y_{t_{0}}$ and $\sup _{t \in\left[t_{0}, T\right]}\|x(t)-y(t)\| \leq \delta$.

\section{B. Neighboring feasible trajectories theorems}

Let $\lambda>0$. Consider the following inward pointing condition:

$$
\left(I P C^{\lambda}\right)\left\{\begin{array}{l}
\forall t \in[0, T], \forall \psi \in \mathcal{K}_{\lambda}, \forall v \in F(t, \psi) \\
\text { such that } \max _{n \in N_{K}^{1}(\psi(0))}\langle n, v\rangle \geq 0, \\
\exists w \in \operatorname{Liminf}_{(s, \phi) \rightarrow(t, \psi)} F(s, \phi) \\
\text { satisfying } \max _{n \in N_{K}^{1}(\psi(0))}\langle n, w-v\rangle<0,
\end{array}\right.
$$

where $\mathcal{K}_{\lambda}$ is defined by (5). Before stating our first NFT theorem, a crucial result is given by the following lemma which shows that $\left(I P C^{\lambda}\right)$ implies an uniform inward pointing condition on a neighborhood of $\mathcal{K}_{\lambda}$.

Lemma 1: Let $\lambda>0$ and assume (H1)-(H4) and $\left(I P C^{\lambda}\right)$. Then $\forall R>0, \exists \rho>0$ and $\eta>0$ such that for every $t \in[0, T], \psi \in\left(\mathcal{K}_{\lambda}+\eta B_{C}\right) \cap R B_{C}$ and for every $v \in F(t, \psi)$ with

$$
\max _{n \in N_{K}^{1}(x), x \in \partial K \cap B(\psi(0), \eta)}\langle n, v\rangle \geq 0
$$


we can find $w \in F(t, \psi)$ satisfying

$$
\left\{\begin{array}{l}
\langle n, w\rangle \leq-\rho \text { and }\langle n, w-v\rangle \leq-\rho \\
\forall n \in N_{K}^{1}(x), \forall x \in \partial K \cap B(\psi(0), \eta) .
\end{array}\right.
$$

The following theorem shows the existence of a feasible $F$-trajectory and provides an estimate of the distance (in the norm of uniform convergence) of this trajectory from a specified $F$-trajectory.

Theorem 3: Assume (H1)-(H3). Let $\tau>0, r_{0}>0$ and $\lambda_{0}>0$ and suppose that, for

$$
\lambda=\max \left\{\lambda_{0},\left(1+\left(1+\lambda_{0} \tau+r_{0}\right) e^{\sigma T}\right) \sigma\right\},
$$

assumptions ( $\mathrm{H} 4)$ and $\left(I P C^{\lambda}\right)$ hold true. Then there exists a constant $C>0$ such that for any $t_{0} \in[0, T]$ and every $F$-trajectory $\hat{x}(\cdot)$ on $\left[t_{0}-\tau, T\right]$ with $\lambda_{0}$-Lipschitz $\hat{x}_{t_{0}}$ and $\hat{x}\left(t_{0}\right) \in K \cap r_{0} B$, and for any $\varepsilon_{0}>0$, we can find a feasible $F$-trajectory on $\left[t_{0}-\tau, T\right]$ satisfying $x_{t_{0}}=\hat{x}_{t_{0}}, x\left(\left(t_{0}, T\right]\right) \subset$ Int $K$ and

$$
\left\|x_{t}-\hat{x}_{t}\right\|_{C} \leq C\left(\max _{t \in\left[t_{0}, T\right]} d_{K}(\hat{x}(t))+\varepsilon_{0}\right) .
$$

Theorem 3 together with Theorem 2 imply that under the inward pointing condition $\left(I P C^{\lambda}\right)$, the set of $F$ trajectories lying in the interior of the constraint set $K$, for $t \in\left(t_{0}, T\right]$ and starting at $\hat{x}_{t_{0}}$, is dense in the set of feasible relaxed $F$-trajectories. This results from the following corollary:

Corollary 1: Under all the assumptions of Theorem 3, for any feasible relaxed $F$-trajectory $\bar{x}(\cdot)$ with $\lambda_{0}$-Lipschitz $\bar{x}_{t_{0}}$ and $\bar{x}\left(t_{0}\right) \in K \cap r_{0} B$, and any $\delta>0$, there exists a feasible F-trajectory $x(\cdot)$ such that $x_{t_{0}}=\bar{x}_{t_{0}}, x\left(\left(t_{0}, T\right]\right) \in$ Int $K$ and $\left\|x_{t}-\bar{x}_{t}\right\|_{C}<\delta$ for all $t \in\left[t_{0}, T\right]$.

Now, assume the relaxed inward pointing condition given by $\left(I P_{r e l}^{\lambda}\right)$. As before, we have the following lemma which is similar to Lemma 1 but in the framework of the relaxed set-valued map.

Lemma 2: Let $\lambda>0$ and assume (H1)-(H4) and $\left(I P_{\text {rel }}^{\lambda}\right)$. Then $\forall R>0, \exists \rho>0$ and $\eta>0$ such that for every $t \in[0, T], \psi \in\left(\mathcal{K}_{\lambda}+\eta B_{C}\right) \cap R B_{C}$ and for every $v \in \operatorname{co} F(t, \psi)$ with

$$
\max _{n \in N_{K}^{1}(x), x \in \partial K \cap B(\psi(0), \eta)}\langle n, v\rangle \geq 0,
$$

we can find $w \in \operatorname{co} F(t, \psi)$ satisfying

$$
\left\{\begin{array}{l}
\langle n, w\rangle \leq-\rho \text { and }\langle n, w-v\rangle \leq-\rho \\
\forall n \in N_{K}^{1}(x), \forall x \in \partial K \cap B(\psi(0), \eta) .
\end{array}\right.
$$

The following theorem is related to Theorem 3, however neither one is contained in another.

Theorem 4: Assume (H1)-(H3). Let $\tau>0, r_{0}>0$ and $\lambda_{0}>0$ and suppose that, for $\lambda$ given by (11), assumptions ( $\mathrm{H} 4)$ and $\left(I P_{\text {rel }}^{\lambda}\right)$ hold true. Then there exists a constant $C>0$ such that for any $t_{0} \in[0, T]$ and every relaxed F-trajectory $\hat{x}(\cdot)$ on $\left[t_{0}-\tau, T\right]$ with $\lambda_{0^{-}}$ Lipschitz $\hat{x}_{t_{0}}$ and $\hat{x}\left(t_{0}\right) \in K \cap r_{0} B$, and for any $\varepsilon_{0}>0$, we can find a relaxed feasible $F$-trajectory on $\left[t_{0}-\tau, T\right]$ satisfying $x_{t_{0}}=\hat{x}_{t_{0}}, x\left(\left(t_{0}, T\right]\right) \subset$ Int $K$ and

$$
\left\|x_{t}-\hat{x}_{t}\right\|_{C} \leq C\left(\max _{t \in\left[t_{0}, T\right]} d_{K}(\hat{x}(t))+\varepsilon_{0}\right) .
$$

The proof of Theorem 4 is a straightforward consequence of Lemma 2, together with Theorem 3 applied with co $F$ instead of $F$. Theorem 4 and the constructive argument of [9, Proof of Lemma 5.2] imply the following Corollary:

Corollary 2: Under all the assumptions of Theorem 4 , for any relaxed feasible $F$-trajectory $\bar{x}(\cdot)$ with $\lambda_{0}$-Lipschitz $\bar{x}_{t_{0}}$ and $\bar{x}\left(t_{0}\right) \in K \cap r_{0} B$, and any $\delta>0$, there exists a feasible $F$-trajectory $x(\cdot)$ such that $x_{t_{0}}=\bar{x}_{t_{0}}, x\left(\left(t_{0}, T\right]\right) \in \operatorname{Int} K$ and $\left\|x_{t}-\bar{x}_{t}\right\|_{C}<\delta$ for all $t \in\left[t_{0}, T\right]$.

C. Neighboring feasible trajectories theorem: constant delay case

Consider the constant-delay differential inclusion

$$
\left\{\begin{aligned}
\dot{x}(t) & \in \mathcal{F}(t, x(t), x(t-\tau)), \quad \text { a.e. } t \in\left[t_{0}, T\right], \\
x_{t_{0}} & =\varphi
\end{aligned}\right.
$$

where $\mathcal{F}:[0, T] \times \mathbb{R}^{n} \times \mathbb{R}^{n} \rightsquigarrow \mathbb{R}^{n}$ is a set-valued map having closed nonempty images and $\varphi \in \mathcal{C}\left([-\tau, 0], \mathbb{R}^{n}\right)$. Let $\lambda>0$. Consider the following inward pointing condition:

$$
\left(I P_{e q}^{\lambda}\right)\left\{\begin{array}{c}
\forall t \in[0, T], \forall x \in \partial K, \forall y \in x+\tau \lambda B, \\
\forall v \in \mathcal{F}(t, x, y) \text { such that } \max _{n \in N_{K}^{1}(x)}\langle n, v\rangle \geq 0, \\
\exists w \in \operatorname{Liminf}_{(s, z, \xi) \rightarrow(t, x, y)} \operatorname{co} \mathcal{F}(s, z, \xi) \\
\text { satisfying } \max _{n \in N_{K}^{1}(x)}\langle n, w-v\rangle<0 .
\end{array}\right.
$$

Assume the following regularity conditions on $\mathcal{F}$ :

(A1) for every $X=(x, y) \in \mathbb{R}^{n} \times \mathbb{R}^{n}$ the set-valued map $\mathcal{F}(\cdot, X)$ is measurable;

(A2) the set-valued map $\mathcal{F}(t, \cdot)$ is locally Lipschitz, i.e. $\forall R>0, \exists \zeta_{R}(\cdot) \in \mathcal{L}^{1}\left(\left[t_{0}, T\right], \mathbb{R}^{+}\right)$such that, for a.e. $t \in\left[t_{0}, T\right]$ and any $X=\left(x_{1}, y_{1}\right), Y=$ $\left(x_{2}, y_{2}\right) \in R B \times R B$

$$
\mathcal{F}(t, X) \subset \mathcal{F}(t, Y)+\zeta_{R}(t)\|X-Y\| B
$$

(A3) there exists $\sigma>0$ such that, for a.e. $t \in\left[t_{0}, T\right]$ and any $X=(x, y) \in \mathbb{R}^{n} \times \mathbb{R}^{n}$

$$
\mathcal{F}(t, X) \subset \sigma(1+|X|) B
$$


(A4) for a given $\lambda>0$, the set-valued map $\mathcal{F}$ is upper semicontinuous on $\left[t_{0}, T\right] \times(\partial K+\tau \lambda B)$.

Theorem 5: Assume (A1)-(A3). Let $\tau>0, r_{0}>0$ and $\lambda_{0}>0$ and suppose that, for $\lambda$ given by (11), assumptions (A4) and $\left(I P_{e q}^{\lambda}\right)$ hold true. Then there exists a constant $C>0$ such that for any $t_{0} \in[0, T]$ and every $\mathcal{F}$-trajectory $\hat{x}(\cdot)$ on $\left[t_{0}-\tau, T\right]$ with $\lambda_{0}$-Lipschitz $\hat{x}_{t_{0}}$ and $\hat{x}\left(t_{0}\right) \in K \cap r_{0} B$, and for any $\varepsilon_{0}>0$, we can find $a$ feasible $\mathcal{F}$-trajectory on $\left[t_{0}-\tau, T\right]$ satisfying $x_{t_{0}}=\hat{x}_{t_{0}}$, $x\left(\left(t_{0}, T\right]\right) \subset \operatorname{Int} K$ and

$$
\left\|x_{t}-\hat{x}_{t}\right\|_{C} \leq C\left(\max _{t \in\left[t_{0}, T\right]} d_{K}(\hat{x}(t))+\varepsilon_{0}\right) .
$$

\section{EXAMPLE: Population DYNAMics}

Here, we present an example that motivated us for this study. It concerns the management of urban pigeon population. In fact, urban pigeon population can reach high densities in cities and disturb the cohabitation with urban citizens. In view of some ecological considerations, this increased population may lead to a citizen dissatisfaction. A model describing the evolution of such population which is subject to some management strategies is proposed in [22], and the urban citizen tolerance formulated as a state constraint. The population dynamics is given by ${ }^{1}$

$$
\begin{aligned}
& \dot{x}_{1}=n\left(x_{2}, u\right) x_{2}-m_{1}\left(x_{1}, u\right) x_{1}-p_{1}\left(x_{1}, u\right) x_{1} \\
& \dot{x}_{2}=-m_{2}\left(x_{2}, u\right) x_{2}+p_{1}\left(x_{1}, u\right) x_{1},
\end{aligned}
$$

where $x_{1}$ and $x_{2}$ denote the size of juvenile and adult pigeon populations and $u$ is the control parameter relative to a management strategy (resource limitation, egg removal, sterilization, capturing). The function $n(\cdot)$ describes the reproduction of adult pigeon; $m_{1}(\cdot)$ and $m_{2}(\cdot)$ describe the mortality of juvenile and adult pigeons. The function $p_{1}(\cdot)$ represents the transfer rate from juvenile to adult class. The urban citizen tolerance is described through the following state constraints set

$$
K= \begin{cases}\underline{M} \leq x_{1}+x_{2} \leq \bar{M}, & \forall t \geq 0, \\ x_{1} \geq 0, \quad x_{2} \geq 0, & \forall t \geq 0,\end{cases}
$$

where $\underline{M}$ and $\bar{M}$ represent the lower (the presence of some pigeons) and upper (not too many pigeons) limits. Thanks to the viability theory, viability kernels describing the existence of efficient management strategies are calculated (see [22] for more details).

The model (16) is not sufficiently precise, because it

\footnotetext{
${ }^{1}$ By abuse of notation, we omit writing explicitly the dependence of $\dot{x}_{i}, x_{i}$, and $u$ on $t$, for $i=1,2$.
}

does not take into account the fact that juvenile pigeons start to reproduce only after becoming adults. This leads naturally to a model with time delay that we describe next. Actually, the transfer from juvenile to adult class is modeled as a function with a delay involving the adult pigeons, taking into account the following observation: the juveniles which born a time $t-\tau$ and survive to time $t$ exit to adult pigeon class, where $\tau$ is the time from birth to maturity. This can be formulated by the following equations (see [1] for more details)

$$
\begin{aligned}
& \dot{x}_{1}=n\left(x_{2}, u\right) x_{2}-m_{1}\left(x_{1}, u\right) x_{1}-p_{2} x_{2}(t-\tau) \\
& \dot{x}_{2}=-m_{2}\left(x_{2}, u\right) x_{2}+p_{2} x_{2}(t-\tau),
\end{aligned}
$$

where $p_{2}$ is the corresponding survival rate of juvenile pigeons born at $\tau$-time ago, which is taken constant in order to simplify the presentation (in fact, it depends on $x_{2}(t-\tau)$ and $\left.u\right)$. Let $x=\left(x_{1}, x_{2}\right)$ and $y=\left(y_{1}, y_{2}\right) \in \mathbb{R}^{2}$. System (17) can be written in the form of (14), where the set-valued map $\mathcal{F}: \mathbb{R}^{2} \times \mathbb{R}^{2} \rightsquigarrow$ $\mathbb{R}^{2}$ is given by

$\mathcal{F}(x, y)=\bigcup_{u \in U}\left(\begin{array}{c}n\left(x_{2}, u\right) x_{2}-m_{1}\left(x_{1}, u\right) x_{1}-p_{2} y_{2} \\ -m_{2}\left(x_{2}, u\right) x_{2}+p_{2} y_{2}\end{array}\right)$.

To extend the study realized in [22] to the time delayed case, Corollary 2 is crucial. Indeed, viability algorithms are conceived for convex maps. In the case of (17), in general, $\mathcal{F}$ is not convex. Knowing that $n(\cdot), m_{1}(\cdot)$ and $m_{2}(\cdot)$ are sufficiently regular then $\mathcal{F}$ fulfills the assumption of Theorem 5. Thus, if condition $\left(I P_{e q}^{\lambda}\right)$ holds true on the boundary of $K$, Corollary 2 guaranties the existence of feasible trajectories for (17), approximating a feasible relaxed trajectory of the convexified problem.

\section{Proofs}

\section{A. Proof of Theorem 1}

We need the following lemma from [14]:

Lemma 3: Let $X$ be a separable Banach space, $\mathcal{G}$ be a set-valued map from $\left[t_{0}, T\right] \times X$ into closed nonempty subsets of $X$ and $z:\left[t_{0}, T\right] \rightarrow X$ be a continuous function such that

1) $\forall x \in X$ the set-valued map $\mathcal{G}(\cdot, x)$ is measurable.

2) $\exists \beta>0, \bar{\zeta}(\cdot) \in \mathcal{L}^{1}\left(\left[t_{0}, T\right], \mathbb{R}^{+}\right)$such that for almost all $t \in\left[t_{0}, T\right]$ the map $\mathcal{G}(t, \cdot)$ is $\bar{\zeta}(t)$-Lipschitzian on $z(t)+\beta B_{X}$, where $B_{X}$ is the closed unit ball in $X$ centered at 0 .

Let $x \in \mathcal{C}\left(\left[t_{0}, T\right], X\right)$ be such that $\|x-z\|_{C} \leq \beta$. Then the set-valued map $t \rightsquigarrow \mathcal{G}(t, x(t))$ is measurable.

In addition to Lemma 3 , the proof of Theorem 1 requires the following two lemmas. The first one states that, for every $x \in \mathcal{C}\left(\left[t_{0}-\tau, T\right], \mathbb{R}^{n}\right)$ taken in a neighborhood 
of the reference trajectory $y$, the map $t \rightsquigarrow F\left(t, x_{t}\right)$ is measurable.

Lemma 4: Let $\beta>0$. Assume (H1), (H2) and let $y$ be as in Theorem 1. Let $x \in \mathcal{C}\left(\left[t_{0}-\tau, T\right], \mathbb{R}^{n}\right)$ be such that $\|x(t)-y(t)\| \leq \beta$, for every $t \in\left[t_{0}-\tau, T\right]$. Then the set-valued map $t \rightsquigarrow F\left(t, x_{t}\right)$ is measurable.

Proof. Since $x$ and $y$ are continuous on $\left[t_{0}-\tau, T\right]$, we can easily prove (see [23, Lemma 2.1] for more details) that $x_{t}$ and $y_{t}$ are also continuous functions of $t$ on $\left[t_{0}, T\right]$. By $(\mathrm{H} 2), F(t, \cdot)$ is $\bar{\zeta}(t)$-Lipschitzian on $y_{t}+\beta B_{C}$ with the Lipschitz constant $\bar{\zeta}(\cdot)=\zeta_{R+\beta}(\cdot)$. Then, by Lemma 3 (with $X=\mathcal{C}\left([-\tau, 0], \mathbb{R}^{n}\right), \mathcal{G}=F$ and $z(\cdot)=y(\cdot+\theta), \theta \in[-\tau, 0])$, we obtain that the set-valued function $\left[t_{0}, T\right] \ni t \rightsquigarrow F\left(t, x_{t}\right)$ is measurable, which concludes the proof.

The following lemma proves that, starting from a reference trajectory $y$, we can construct a sequence $\left(x_{n}\right)_{n \geq 0}$ in $\mathcal{C}\left(\left[t_{0}-\tau, T\right], \mathbb{R}^{n}\right)$ approximating a solution of (6)-(7).

Lemma 5: Let $\beta>0$ and $\delta_{0} \geq 0$. Assume (H1), (H2) and let $y, \gamma_{1}, \gamma_{2}, \gamma_{3}$ be as in Theorem 1. If $\gamma_{3}(T)<\beta$, then for any $\varphi \in \mathcal{C}\left([-\tau, 0], \mathbb{R}^{n}\right)$ with $\left\|\varphi-y_{t_{0}}\right\|_{C} \leq \delta_{0}$ there exist sequences $x_{n} \in \mathcal{C}\left(\left[t_{0}-\tau, T\right], \mathbb{R}^{n}\right)$ and $f_{n} \in \mathcal{L}^{1}\left(\left[t_{0}, T\right], \mathbb{R}^{n}\right)$, for $n \geq 0$, such that

$$
\begin{array}{r}
x_{0, t}=y_{t}, \quad f_{0}=\dot{y}, \quad t \in\left[t_{0}, T\right], \\
\left\|f_{1}(t)-f_{0}(t)\right\|=\gamma_{1}(t), \quad \text { a.e. } t \in\left[t_{0}, T\right]
\end{array}
$$

and for $n \geq 1$

$$
\begin{aligned}
& \left\{\begin{array}{l}
x_{n}(t)=\varphi(0)+\int_{t_{0}}^{t} f_{n}(s) d s, \quad t \in\left[t_{0}, T\right], \\
x_{n, t_{0}}=\varphi
\end{array}\right. \\
& f_{n}(t) \in F\left(t, x_{n-1, t}\right), \quad t \in\left[t_{0}, T\right],
\end{aligned}
$$

with

$$
\left\|f_{n+1}(t)-f_{n}(t)\right\| \leq \zeta_{R+\beta}(t)\left\|x_{n, t}-x_{n-1, t}\right\|_{C},
$$

for almost every $t \in\left[t_{0}, T\right]$.

Proof. By Lemma 4, the set-valued map $t \rightsquigarrow F\left(t, y_{t}\right)$ is measurable. Since the function $t \rightarrow \gamma_{1}(t)$ is measurable (see, [14, Lemma 1.5]), the set-valued map $U_{1}$ defined by

$$
U_{1}(t):=\left\{v \in F\left(t, y_{t}\right):\left\|v-f_{0}(t)\right\|=\gamma_{1}(t)\right\}
$$

is measurable (see, e.g., [3]). Hence, by the measurable selection theorem the set-valued map $U_{1}$ admits a measurable selection $f_{1}:\left[t_{0}, T\right] \mapsto \mathbb{R}^{n}$. From the definition of $U_{1}$, we have $f_{1}(t) \in F\left(t, y_{t}\right)$ for $t \in\left[t_{0}, T\right]$ and

$$
\left\|f_{1}(t)-f_{0}(t)\right\|=\gamma_{1}(t) \quad \text { a.e. } t \in\left[t_{0}, T\right] .
$$

Let $\varphi \in \mathcal{C}\left([-\tau, 0], \mathbb{R}^{n}\right)$ be such that $\left\|\varphi-y_{t_{0}}\right\|_{C} \leq \delta_{0}$ and define $x_{1} \in \mathcal{C}\left(\left[t_{0}-\tau, T\right], \mathbb{R}^{n}\right)$ by

$$
\left\{\begin{aligned}
x_{1}(t) & =\varphi(0)+\int_{t_{0}}^{t} f_{1}(s) d s, & & t \in\left[t_{0}, T\right], \\
x_{1}\left(t_{0}+\theta\right) & =\varphi(\theta), & & \theta \in[-\tau, 0] .
\end{aligned}\right.
$$

Observe that

$$
\left\|x_{1, t}-y_{t}\right\| \leq \delta_{0}+\int_{t_{0}}^{t} \gamma_{1}(s) d s, \quad t \in\left[t_{0}, T\right]
$$

Indeed, for $\theta \in[-\tau, 0]$ and $t \in\left[t_{0}, T\right]$ such that $t+\theta \geq t_{0}$, we have

$$
\begin{aligned}
& \left\|x_{1}(t+\theta)-y(t+\theta)\right\| \\
& \leq\left\|\varphi(0)-y\left(t_{0}\right)\right\|+\left\|\int_{t_{0}}^{t+\theta} f_{1}(s)-\dot{y}(s) d s\right\| \\
& \leq\left\|\varphi-y_{t_{0}}\right\|_{C}+\int_{t_{0}}^{t+\theta} \gamma_{1}(s) d s \\
& \leq \delta_{0}+\int_{t_{0}}^{t} \gamma_{1}(s) d s .
\end{aligned}
$$

In the case when $t+\theta<t_{0}$, we have $\left\|x_{1}(t+\theta)-y(t+\theta)\right\|=\left\|\varphi-y_{t_{0}}\right\|_{C} \leq \delta_{0}$.

With $x_{1}$, we associate the set-valued map $\left[t_{0}, T\right] \ni t \rightsquigarrow U_{2}(t)$ defined by $U_{2}(t):=\{v \in$ $\left.F\left(t, x_{1, t}\right):\left\|v-\dot{x}_{1}(t)\right\|=d_{F\left(t, x_{1, t}\right)}\left(\dot{x}_{1}(t)\right)\right\}$. By (24), $\left\|x_{1, t}-y_{t}\right\|_{C} \leq \beta$ and, since $F(t, \cdot)$ is $\zeta_{R+\beta}$-Lipschitz on $y_{t}+\beta B_{C}$, we deduce (using the same arguments as before) the existence of a measurable selection $f_{2}:\left[t_{0}, T\right] \mapsto \mathbb{R}^{n}$ of $U_{2}$ such that $\left\|f_{2}(t)-f_{1}(t)\right\| \leq \zeta_{R+\beta}(t)\left\|x_{1, t}-x_{0, t}\right\|_{C}$, for almost every $t \in\left[t_{0}, T\right]$. Then, we conclude that (20)-(22) hold true for $n=1$.

Assume that we already have constructed $x_{n} \in \mathcal{C}\left(\left[t_{0}-\tau, T\right], \mathbb{R}^{n}\right)$ and $f_{n} \in \mathcal{L}^{1}\left(\left[t_{0}, T\right], \mathbb{R}^{n}\right)$, for $n=1, \cdots, N$, verifying (20), (21) and (22). Before extending to $n=N+1$, we prove that the constructed sequence $x_{n}$ verifies the following:

Claim 1: $\left\|x_{n, t}-y_{t}\right\|_{C} \leq \beta, \forall t \in\left[t_{0}, T\right], \forall n=$ $1, \cdots, N$.

In fact, for $n=1$, the claim follows directly from (24). For $n \geq 2$, (22) implies the following inequalities:

$$
\begin{aligned}
& \left\|x_{n, t}-x_{n-1, t}\right\|_{C} \leq \int_{t_{0}}^{t}\left\|f_{n}\left(s_{1}\right)-f_{n-1}\left(s_{1}\right)\right\| d s_{1} \\
& \leq \int_{t_{0}}^{t} \zeta_{R+\beta}\left(s_{1}\right)\left\|x_{n-1, s_{1}}-x_{n-2, s_{1}}\right\|_{C} d s_{1},
\end{aligned}
$$


which can be repeated recursively (see the proof of [14, Theorem 1.2] for more details) to obtain the following property:

$$
\left\|x_{n, t}-x_{n-1, t}\right\|_{C} \leq\left(\delta_{0}+\int_{t_{0}}^{t} \gamma_{1}(s) d s\right) \frac{\left[\ln \left(\gamma_{2}(t)\right)\right]^{n}}{n !},
$$

for every $n \in\{2, \cdots, N\}$. From (24) and the last inequality, we get (see the proof of [14, Theorem 1.2] for more details)

$$
\begin{aligned}
\left\|x_{N, t}-y_{t}\right\|_{C} & \leq \sum_{i=1}^{N}\left\|x_{i, t}-x_{i-1, t}\right\|_{C} \\
& \leq \gamma_{3}(t) \leq \beta
\end{aligned}
$$

Again, define the set-valued map $\left[t_{0}, T\right] \ni t \rightsquigarrow U_{N+1}(t)$ by $U_{N+1}(t):=\left\{v \in F\left(t, x_{N, t}\right):\left\|v-\dot{x}_{N}(t)\right\|=\right.$ $\left.d_{F\left(t, x_{N, t}\right)}\left(\dot{x}_{N}(t)\right)\right\}$. Knowing that $\left\|x_{N, t}-y_{t}\right\|_{C} \leq \beta$ and using the same reasoning as before, we deduce the existence of $f_{N+1}:\left[t_{0}, T\right] \mapsto \mathbb{R}^{n}$, a measurable selection of $U_{N+1}$, such that $\left\|f_{N+1}(t)-f_{N}(t)\right\| \leq \zeta_{R+\beta}(t)\left\|x_{N, t}-x_{N-1, t}\right\|_{C}$, for almost every $t \in\left[t_{0}, T\right]$. The function $x_{N+1}$, associated to $f_{N+1}$, is defined by (20), for $n=N+1$.

Proof of Theorem 1. By (25), for each $t \in\left[t_{0}, T\right]$, the sequence $\left\{x_{n, t}\right\}$ is Cauchy in the Banach space $\mathcal{C}\left([-\tau, 0], \mathbb{R}^{n}\right)$. Thus, for each $t \in\left[t_{0}, T\right]$ we may define $x_{t} \in \mathcal{C}\left([-\tau, 0], \mathbb{R}^{n}\right)$ as the limit of $x_{n, t}$. In addition, by (22), for almost every $t \in\left[t_{0}, T\right]$ the sequence $\left\{f_{n}(t)\right\}$ is Cauchy in $\mathbb{R}^{n}$. Furthermore, from (22) and (23), it follows that for $n \geq 1$

$$
\begin{aligned}
& \left\|f_{n}(t)-\dot{y}(t)\right\| \leq \sum_{i=0}^{n-1}\left\|f_{i+1}(t)-f_{i}(t)\right\| \\
& \leq \gamma_{1}(t)+\zeta_{R+\beta}(t) \sum_{i=1}^{n-1}\left\|x_{i, t}-x_{i-1, t}\right\|_{C} \\
& \leq \gamma_{1}(t)+\zeta_{R+\beta}(t) \gamma_{3}(t) \quad \text { a.e. } t \in\left[t_{0}, T\right],
\end{aligned}
$$

from which we conclude that the sequence $\left\{f_{n}\right\}$ is integrably bounded. Thus we may define $f \in \mathcal{L}^{1}\left(\left[t_{0}, T\right], \mathbb{R}^{n}\right)$ by $f(t)=\lim _{n \rightarrow+\infty} f_{n}(t)$. By arguments similar to [14, Theorem 1.2], we obtain that $x(\cdot)$ is an $F$-Trajectory satisfying $\dot{x}(t)=f(t)$ a.e. in $\left[t_{0}, T\right]$. Passing to the limits in (26) and (27) yields the desired estimations on $x$ and $\dot{x}$.

\section{B. Proof of Theorem 2}

Fix $\delta>0$ and let $R:=\max _{t \in\left[t_{0}-\tau, T\right]}\|y(t)\|$. It is not restrictive to assume that $t_{0}=0$ and $\int_{0}^{T} \zeta_{R+\delta}(t) d t>0$.
Choose any positive $\alpha$ such that

$$
\alpha<\min \left\{\frac{\delta}{2}, \frac{\delta}{2 \gamma_{2}(T) \int_{0}^{T} \zeta_{R+\delta}(t) d t}\right\},
$$

where $\gamma_{2}(\cdot)$ is as in Theorem 1 , with $\beta=\delta$. Let $n \geq 1$ be so large such that $\sigma(1+R) / n<\alpha / 2$, where $\sigma$ is given by (H3). Let $I_{j}$ be the interval $\left[(j-1) \frac{T}{n}, j \frac{T}{n}\right]$, for $j=1, \cdots, n$. By Lemma 4 , the set-valued map $t \rightsquigarrow F\left(t, y_{t}\right)$ is measurable. In addition, by (H3), $t \rightsquigarrow F\left(t, y_{t}\right)$ is integrably bounded because for almost every $t \in[0, T], F\left(t, y_{t}\right) \subset \sigma(1+R) B$. Then, by Aumann's Theorem [5], there exists a measurable selection $f_{j}(t) \in F\left(t, y_{t}\right)$ such that

$$
\int_{I_{j}} f_{j}(t) d t=\int_{I_{j}} \dot{y}(t) d t, \quad j=1, \cdots, n .
$$

Let $f$ be the function which is equal to $f_{j}$ on $I_{j}$, and define the continuous function $z:[-\tau, T] \rightarrow \mathbb{R}^{n}$ by

$$
\left\{\begin{aligned}
z(t) & =y(0)+\int_{0}^{t} f(s) d s, \quad t \in[0, T], \\
z_{0} & =y_{0} .
\end{aligned}\right.
$$

Observe that

$$
\left\|z_{t}-y_{t}\right\|_{C}<\alpha, \quad \forall t \in[0, T] .
$$

Indeed, for every $t \in[0, T]$ and every $\theta \in[-\tau, 0]$ such that $t+\theta \geq 0$, there exists $j \in\{1, \cdots, n\}$ for which $t+\theta \in I_{j}$ and

$$
\begin{aligned}
\|z(t+\theta)-y(t+\theta)\| & =\left\|\int_{0}^{t+\theta}(f(s)-\dot{y}(s)) d s\right\| \\
& \leq \int_{I_{j}}\|f(s)-\dot{y}(s)\| d s<\alpha .
\end{aligned}
$$

If $t+\theta<0$, then $\|z(t+\theta)-y(t+\theta)\|=0$.

Since for almost every $t \in[0, T], F(t, \cdot)$ is $\zeta_{R+\delta}(t)$ Lipschitz on $y_{t}+\delta B_{C}$, we obtain

$$
\begin{aligned}
d_{F\left(t, z_{t}\right)}(\dot{z}(t)) \leq & \sup \left\{d_{F\left(t, z_{t}\right)}(\xi): \xi \in F\left(t, y_{t}\right)\right\} \\
& +d_{F\left(t, y_{t}\right)}(\dot{z}(t)) \\
\leq & \zeta_{R+\delta}(t)\left\|z_{t}-y_{t}\right\|_{C} \leq \alpha \zeta_{R+\delta}(t) .
\end{aligned}
$$

Inequality (29) together with (28) imply that $\gamma_{2}(T) \int_{0}^{T} \alpha \zeta_{R+\delta}(t) d t<\delta / 2$. Then, by Theorem 1 applied with $\beta=\delta$ and $\delta_{0}=0$, there exists a trajectory $x$ of (6) satisfying $x_{0}=z_{0}=y_{0}$ and

$$
\left\|x_{t}-z_{t}\right\|_{C} \leq \gamma_{2}(T) \int_{0}^{T} \alpha \zeta_{R+\delta}(s) d s<\frac{\delta}{2} .
$$

Finally, we obtain

$$
\left\|x_{t}-y_{t}\right\|_{C} \leq\left\|x_{t}-z_{t}\right\|_{C}+\left\|z_{t}-y_{t}\right\|_{C}<\frac{\delta}{2}+\frac{\delta}{2}=\delta,
$$

which concludes the proof. 


\section{Proof of Lemma 1.}

We proceed in three steps.

Step 1. Let $R>0, \bar{t} \in[0, T]$ and $\bar{\psi} \in \mathcal{K}_{\lambda} \cap R B_{C}$ be fixed. Knowing that $F$ is locally bounded and using exactly the same argument as [17, Lemma 3.5], we prove the existence of $\rho_{\bar{t}, \bar{\psi}}>0$ such that for all $v \in F(\bar{t}, \bar{\psi})$ with $\max _{n \in N_{K}^{1}(\bar{\psi}(0))}\langle n, v\rangle \geq 0$, there exists $w \in \operatorname{Liminf}_{(s, \phi) \rightarrow(\bar{t}, \bar{\psi})} F(s, \phi)$ satisfying

$$
\max \left\{\langle n, w\rangle,\langle n, w-v\rangle \mid n \in N_{K}^{1}(\bar{\psi}(0))\right\} \leq-2 \rho_{\bar{t}, \bar{\psi}} .
$$

Step 2. We show the existence of $\eta_{\bar{t}, \bar{\psi}}>0$ such that for every $t \in B\left(\bar{t}, \eta_{\bar{t}, \bar{\psi}}\right)$, for every $\psi \in \mathcal{K}_{\lambda} \cap B_{C}\left(\bar{\psi}, \eta_{\bar{t}, \bar{\psi}}\right)+$ $\eta_{\bar{t}, \bar{\psi}} B_{C}$ and for every $v \in F(t, \psi)$ with

$$
\max _{n \in N_{K}^{1}(x), x \in \partial K \cap B\left(\psi(0), \eta_{\bar{t}, \bar{\psi}}\right)}\langle n, v\rangle \geq 0,
$$

there exists $w \in F(t, \psi)$ satisfying

$$
\left\{\begin{array}{l}
\langle n, w\rangle \leq-\rho_{\bar{t}, \bar{\psi}} \text { and }\langle n, w-v\rangle \leq-\rho_{\bar{t}, \bar{\psi}} \\
\forall n \in N_{K}^{1}(x), \forall x \in \partial K \cap B\left(\psi(0), \eta_{\bar{t}, \bar{\psi}}\right) .
\end{array}\right.
$$

Suppose by contradiction that there exist $t_{i} \rightarrow \bar{t}, \psi_{i} \rightarrow \bar{\psi}$ in $\mathcal{C}\left([-\tau, 0], \mathbb{R}^{n}\right), v_{i} \in F\left(t_{i}, \psi_{i}\right), x_{i} \rightarrow_{\partial K} \bar{\psi}(0)$ and $n_{i} \in N_{K}^{1}\left(x_{i}\right)$ such that $\left\langle n_{i}, v_{i}\right\rangle \geq 0$ and for every $w_{i} \in F\left(t, \psi_{i}\right)$ we can find $x_{i}^{\prime} \rightarrow_{\partial K} \bar{\psi}(0), n_{i}^{\prime} \in N_{K}^{1}\left(x_{i}^{\prime}\right)$ satisfying

$$
\left\langle n_{i}^{\prime}, w_{i}\right\rangle \vee\left\langle n_{i}^{\prime}, w_{i}-v_{i}\right\rangle>-\rho_{\bar{t}, \bar{\psi}}
$$

Since $F$ is upper semicontinuous at every point of $[0, T] \times \mathcal{K}_{\lambda}$, taking subsequences and keeping the same notations we may assume that $v_{i}$ converge to some $v \in F(\bar{t}, \bar{\psi}), n_{i} \rightarrow n$ and $n_{i}^{\prime} \rightarrow n^{\prime}$. Since the map $x \rightsquigarrow N_{K}^{1}(x)$ is upper semicontinuous, we have $n, n^{\prime} \in N_{K}^{1}(\bar{\psi}(0))$ and $\langle n, v\rangle \geq 0$. Then $\max _{n \in N_{K}^{1}(\bar{\psi}(0))}\langle n, v\rangle \geq 0$. Consider $w$ as in (30) corresponding to this $v$ and let $w_{i} \in F\left(t, \psi_{i}\right)$ be such that $w_{i} \rightarrow w$. From (32) we deduce that $\left\langle n^{\prime}, w\right\rangle \vee\left\langle n^{\prime}, w-v\right\rangle \geq-\rho_{\bar{t}, \bar{\psi}}$, contradicting the choice of $w$.

Step 3. Consider a covering of $[0, T] \times\left(\mathcal{K}_{\lambda} \cap 2 R B_{C}\right)$ by the open balls $\stackrel{\circ}{B}\left((\bar{t}, \bar{\psi}), \eta_{\bar{t}, \bar{\psi}}\right)$ satisfying the following requirement:

$$
\begin{gathered}
{[0, T] \times\left(\mathcal{K}_{\lambda} \cap 2 R B_{C}\right) \subset} \\
\bigcup_{(\bar{t}, \bar{\psi}) \in[0, T] \times \mathcal{K}_{\lambda} \cap 2 R B_{C}} \stackrel{\circ}{B}\left((\bar{t}, \bar{\psi}), \eta_{\bar{t}, \bar{\psi}}\right)
\end{gathered}
$$

such that for every $t \in B\left(\bar{t}, \eta_{\bar{t}, \bar{\psi}}\right)$, for every $\psi \in \mathcal{K}_{\lambda} \cap B_{C}\left(\bar{\psi}, \eta_{\bar{t}, \bar{\psi}}\right)+\eta_{\bar{t}, \bar{\psi}} B_{C}$ and for every $v \in F(t, \psi)$ with

$$
\max _{n \in N_{K}^{1}(x), x \in \partial K \cap B\left(\psi(0), \eta_{\bar{t}, \bar{\psi}}\right)}\langle n, v\rangle \geq 0,
$$

there exists $w \in F(t, \psi)$ satisfying (31).

We claim that the set $\mathcal{K}_{\lambda} \cap 2 R B_{C}$ is compact. Indeed, Thanks to Ascoli's Theorem, we know that a subset of $\mathcal{C}\left([-\tau, 0], \mathbb{R}^{n}\right)$ is compact if and only if it is closed, bounded, and equicontinuous. The set $\mathcal{K}_{\lambda}$ is closed (the uniform limit of $\lambda$-Lipschitz functions is $\lambda$-Lipschitz) and equicontinuous (by assumption). The boundedness follows from the fact that for all $\theta \in[-\tau, 0]$

$$
\|\psi(\theta)\| \leq\|\psi(\theta)-\psi(0)\|+\|\psi(0)\| \leq \lambda \tau+R .
$$

Now, consider a finite subcovering

$$
[0, T] \times\left(\mathcal{K}_{\lambda} \cap 2 R B_{C}\right) \subset \bigcup_{i=1 \cdots, N} \stackrel{\circ}{B}\left(\left(t_{i}, \psi_{i}\right), \eta_{t_{i}, \psi_{i}}\right) .
$$

Then, for $\rho=\min \left\{\rho_{t_{i}, \psi_{i}}, i=1 \cdots, N\right\}$, for some $0<$ $\eta<\min \left\{R, \eta_{t_{i}, \psi_{i}}, i=1 \cdots, N\right\}$ and for all $(t, \psi) \in$ $[0, T] \times\left(\mathcal{K}_{\lambda}+\eta B_{C}\right) \cap R B_{C}$, there exists $1 \leq i \leq N$ such that $(t, \psi) \in B\left(t_{i}, \eta_{t_{i}, \psi_{i}}\right) \times\left(\mathcal{K}_{\lambda} \cap B_{C}\left(\psi_{i}, \eta_{t_{i}, \psi_{i}}\right)+\right.$ $\left.\eta_{t_{i}, \psi_{i}} B_{C}\right)$. This complete the proof.

\section{Proof of Theorem 3.}

The proof is inspired by the construction proposed in [16].

Lemma 6: Assume (H1)-(H3). Let $\tau>0, r>0$ and $\lambda_{0}>0$ and suppose that, for $\lambda$ given by (11), assumptions ( $\mathrm{H} 4)$ and $\left(I P C^{\lambda}\right)$ hold true. Then there exist positive constants $\delta$ and $c$ such that for every $\bar{t} \in[0, T]$ and every F-trajectory $\hat{x}(\cdot)$ on $[\bar{t}-\tau, T]$ with $\lambda_{0}$-Lipschitz $\hat{x}_{\bar{t}}$ and $\hat{x}(\bar{t}) \in K \cap r B$, and for any $\varepsilon>0$, we can find an F-trajectory on $[\bar{t}-\tau, T]$ satisfying

$$
\left\{\begin{array}{l}
x_{\bar{t}}=\hat{x}_{\bar{t}}, \\
x(t) \in \operatorname{Int} K, \quad \forall t \in(\bar{t},(\bar{t}+\delta) \wedge T] \\
\left\|x_{t}-\hat{x}_{t}\right\|_{C} \leq c \max _{t \in[\bar{t}, T]} d_{K}(\hat{x}(t))+\varepsilon .
\end{array}\right.
$$

Proof. Let $R:=\left(1+\lambda_{0} \tau+r\right) e^{\sigma T}, \tilde{R}:=(1+R) \sigma$ and $\bar{R}:=2 \tilde{R} T+R$, where $\sigma$ is as in (H3). Fix $\bar{t} \in[0, T]$ and an $F$-trajectory $\hat{x}(\cdot)$ on $[\bar{t}-\tau, T]$ such that $\hat{x}_{\bar{t}}$ is $\lambda_{0^{-}}$ Lipschitz and $\hat{x}(\bar{t}) \in K \cap r B$. Let $\delta>0$ and $0<\beta<\rho$ be such that $\delta<\frac{\eta}{4 \sigma(1+R)}$ (where $\rho$ and $\eta$ are given by Lemma 1) and

$$
\nu(\delta):=2 \tilde{R} \exp \left(\int_{\bar{t}}^{T} \zeta_{\bar{R}+\beta}(s) d s\right) \omega_{\zeta_{\bar{R}}}(\delta)<\beta,
$$

where $\zeta_{\bar{R}}(\cdot)$ and $\zeta_{\bar{R}+\beta}(\cdot)$ are as in $(\mathrm{H} 2)$, and $\omega_{\zeta_{\bar{R}}}(\cdot)$ is the modulus of continuity of the map $t \mapsto \int_{\bar{t}}^{t} \zeta_{\bar{R}}(s) d s$. Consider $\Gamma>0$ such that $\Gamma(\rho-\nu(\delta))>1$ and call $\bar{\delta}:=(\bar{t}+\delta) \wedge T$. 
We proceed in four steps.

Step 1 . We have $\left\|\hat{x}_{t}\right\|_{C} \leq R$ for every $t \in[\bar{t}, T]$. Indeed, for $t \in[\bar{t}, T]$ and $\theta \in[-\tau, 0]$, we have

$$
\hat{x}(t+\theta)= \begin{cases}\hat{x}(\bar{t})+\int_{\bar{t}}^{t+\theta} \dot{\hat{x}}(s) d s, & \text { if } t+\theta \geq \bar{t} \\ \hat{x}_{\bar{t}}(t+\theta-\bar{t}), & \text { if } t+\theta<\bar{t} .\end{cases}
$$

Then,

$$
\|\hat{x}(t+\theta)\| \leq\left\|\hat{x}_{\bar{t}}\right\|_{C}+\int_{\bar{t}}^{\max \{\bar{t}, t+\theta\}} \sigma\left(1+\left\|\hat{x}_{s}\right\|_{C}\right) d s .
$$

Hence

$$
\left\|\hat{x}_{t}\right\|_{C} \leq\left\|\hat{x}_{\bar{t}}\right\|_{C}+\int_{\bar{t}}^{\max \{\bar{t}, t+\theta\}} \sigma\left(1+\left\|\hat{x}_{s}\right\|_{C}\right) d s .
$$

Thanks to Gronwall's Lemma, we can easily verify that for any $t \in[\bar{t}, T]$

$$
\left\|\hat{x}_{t}\right\|_{C} \leq\left(1+\left\|\hat{x}_{\bar{t}}\right\|_{C}\right) e^{\sigma(t-\bar{t})},
$$

from which conclude that $\left\|\hat{x}_{t}\right\|_{C} \leq R$ for every $t \in[\bar{t}, T]$.

Step 2. If $\tilde{d}_{K}(\hat{x}(\bar{t}))<-\frac{\eta}{4}$, then $x(\cdot)=\hat{x}(\cdot)$ satisfies our lemma. Indeed, if $\tilde{d}_{K}(\hat{x}(\bar{t}))<-\frac{\eta}{4}$, then for all $t \in[\bar{t}, \bar{\delta}]$, we have

$$
\begin{aligned}
& \tilde{d}_{K}(\hat{x}(t)) \leq \tilde{d}_{K}(\hat{x}(\bar{t}))+\left|\tilde{d}_{K}(\hat{x}(t))-\tilde{d}_{K}(\hat{x}(\bar{t}))\right| \\
& <-\frac{\eta}{4}+\|\hat{x}(t)-\hat{x}(\bar{t})\| \leq-\frac{\eta}{4}+\int_{\bar{t}}^{t}\|\dot{\hat{x}}(s)\| d s \\
& <-\frac{\eta}{4}+(1+R) \sigma \delta<0,
\end{aligned}
$$

and $x(\cdot)=\hat{x}(\cdot)$ is as required.

Step 3. If $-\frac{\eta}{4} \leq \tilde{d}_{K}(\hat{x}(\bar{t})) \leq 0$, we define the measurable set

$$
\begin{gathered}
\mathcal{S}:=\left\{s \in[\bar{t}, \bar{\delta}]: \exists x \in \partial K \cap B(\hat{x}(s), \eta), n \in N_{K}^{1}(x),\right. \\
\langle n, \hat{\hat{x}}(s)\rangle \geq 0\} .
\end{gathered}
$$

Fix any $\varepsilon>0$ and $\varepsilon^{\prime}>0$ such that

$$
0<\varepsilon^{\prime}<\frac{\varepsilon}{2 \tilde{R}\left[1+\exp \left(\int_{0}^{T} \zeta_{\bar{R}+\beta}(s) d s\right) \int_{0}^{T} \zeta_{\bar{R}}(s) d s\right]},
$$

and let $\kappa \in[\bar{t}, \bar{\delta}]$ be defined as follows:

- If $\mu(\mathcal{S}) \leq \Gamma \max _{s \in[\bar{t}, T]} d_{K}(\hat{x}(s))+\varepsilon^{\prime}$ then set $\kappa=\bar{\delta}$.

- If $\mu(\mathcal{S})>\Gamma \max _{s \in[\bar{t}, T]} d_{K}(\hat{x}(s))+\varepsilon^{\prime}$ then take $\kappa$ be the smallest number in $[\bar{t}, \bar{\delta}]$ such that

$$
\mu(\mathcal{S} \cap[\bar{t}, \kappa])=\Gamma \max _{s \in[\bar{t}, T]} d_{K}(\hat{x}(s))+\varepsilon^{\prime} .
$$

For each $s \in \mathcal{S}$, we have $\hat{x}_{s} \in\left(\mathcal{K}_{\lambda}+\eta B_{C}\right) \cap R B_{C}$. Indeed, let $z \in \partial K$ be such that $\|z-\hat{x}(s)\|=d_{K}(\hat{x}(s))$. Let us define the function $\psi \in \mathcal{C}\left([-\tau, 0], \mathbb{R}^{n}\right)$ by

$$
\psi(\theta)=\hat{x}(s+\theta)-\hat{x}(s)+z, \quad \theta \in[-\tau, 0] .
$$

The function $\psi$ belongs to $\mathcal{K}_{\lambda}$. In addition, we have

$$
\begin{aligned}
& \left\|\hat{x}_{s}(\theta)-\psi(\theta)\right\|=\|\hat{x}(s)-z\|=d_{K}(\hat{x}(s)) \\
& \leq d_{K}(\hat{x}(\bar{t}))+\left|d_{K}(\hat{x}(s))-d_{K}(\hat{x}(\bar{t}))\right|<\eta .
\end{aligned}
$$

Then $\hat{x}_{s} \in\left(\mathcal{K}_{\lambda}+\eta B_{C}\right) \cap R B_{C}$. Thanks to Lemma 1, for each $s \in \mathcal{S}$, we can find $w \in F\left(s, \hat{x}_{s}\right)$ satisfying (10). By the measurable selection theorem [3], there exists a measurable function $w: \mathcal{S} \rightarrow \mathbb{R}^{n}$ such that $w(s) \in F\left(s, \hat{x}_{s}\right)$, and for a.e. $s \in \mathcal{S}$

$$
\left\{\begin{array}{l}
\langle n, w(s)\rangle \leq-\rho, \text { and }\langle n, w(s)-\dot{\hat{x}}(s)\rangle \leq-\rho \\
\forall x \in \partial K \cap B(\hat{x}(s), \eta), \forall n \in N_{K}^{1}(x) .
\end{array}\right.
$$

Define the absolutely continuous function $y:[\bar{t}-\tau, T] \mapsto$ $\mathbb{R}^{n}$ by $y_{\bar{t}}=\hat{x}_{\bar{t}}$ and

$$
\dot{y}(t):=\left\{\begin{array}{ccl}
w(t) & \text { if } \quad t \in \mathcal{S} \cap[\bar{t}, \kappa] \\
\dot{\hat{x}}(t) & \text { if } \quad t \in([\bar{t}, T] \backslash \mathcal{S}) \cap[\bar{t}, \kappa] .
\end{array}\right.
$$

For $t \in[\bar{t}, T]$ and $\theta \in[-\tau, 0]$, we have

$$
\begin{aligned}
& \left\|y_{t}(\theta)-\hat{x}_{t}(\theta)\right\| \leq\left\|\int_{\bar{t}}^{\max \{\bar{t}, t+\theta\}}(\dot{y}(s)-\dot{\hat{x}}(s)) d s\right\| \\
& \leq \int_{\bar{t}}^{t}\|\dot{y}(s)-\dot{\hat{x}}(s)\| d s=\int_{\mathcal{S} \cap[\bar{t}, t \wedge \kappa]}\|w(s)-\dot{\hat{x}}(s)\| d s \\
& \leq 2 \tilde{R} \mu(\mathcal{S} \cap[\bar{t}, t \wedge \kappa])
\end{aligned}
$$

implying that,

$$
\left\|y_{t}-\hat{x}_{t}\right\|_{C} \leq 2 \tilde{R} \mu(\mathcal{S} \cap[\bar{t}, t \wedge \kappa]) .
$$

Moreover, for a.e. $t \in[\bar{t}, T]$,

$$
\begin{gathered}
d_{F\left(t, y_{t}\right)}(\dot{y}(t)) \leq \\
\sup \left\{d_{F\left(t, \hat{x}_{t}\right)}(\xi): \xi \in F\left(t, y_{t}\right)\right\}+d_{F\left(t, \hat{x}_{t}\right)}(\dot{y}(t)) \\
\leq \zeta_{\bar{R}}(t)\left\|y_{t}-\hat{x}_{t}\right\| \leq 2 \tilde{R} \mu(\mathcal{S} \cap[\bar{t}, t \wedge \kappa]) \zeta_{\bar{R}}(t) .
\end{gathered}
$$

Hence, thanks to Theorem 1 applied with $\delta_{0}=0$, there exists an $F$-trajectory $x(\cdot)$ on $[\bar{t}-\tau, T]$ such that $x_{\bar{t}}=y_{\bar{t}}$ and for every $t \in[\bar{t}, T]$

$$
\begin{gathered}
\left\|x_{t}-y_{t}\right\|_{C} \leq \\
2 \tilde{R} \exp \left(\int_{0}^{T} \zeta_{\bar{R}+\beta}(s) d s\right) \omega_{\zeta_{\bar{R}}}(|t-\bar{t}|) \mu(\mathcal{S} \cap[\bar{t}, t \wedge \kappa]) .
\end{gathered}
$$

By the definition of $\kappa$, the inequality (36) and the triangle inequality, we have

$$
\begin{aligned}
\left\|x_{t}-\hat{x}_{t}\right\|_{C} & \leq \frac{\varepsilon}{\varepsilon^{\prime}} \mu(\mathcal{S} \cap[\bar{t}, \kappa]) \\
& \leq c \max _{t \in[\bar{t}, T]} d_{K}(\hat{x}(t))+\varepsilon,
\end{aligned}
$$

for a constant $c$ independent from $\bar{t}$ and $\hat{x}(\cdot)$.

Step 4. We show next that $\{x(t): t \in(\bar{t}, \bar{\delta}]\} \subset \operatorname{Int} K$. We distinguish two different cases: 
Case 1. $t \in(\bar{t}, \kappa]$ : The mean-value theorem (see, e.g., [11, Theorem 2.3.7]), affirms the existence of some $z(t) \in[y(\bar{t}), y(t)]$ and $\xi(t) \in \partial \tilde{d}_{K}(z(t))$, such that

$$
\tilde{d}_{K}(y(t))=\tilde{d}_{K}(y(\bar{t}))+\langle\xi(t), y(t)-y(\bar{t})\rangle .
$$

Then,

$$
\begin{aligned}
& \tilde{d}_{K}(x(t)) \leq \tilde{d}_{K}(y(t))+\|x(t)-y(t)\| \\
& \leq \tilde{d}_{K}(y(t))+\nu(\delta) \mu(\mathcal{S} \cap[\bar{t}, t \wedge \kappa]) \\
& \leq \tilde{d}_{K}(y(\bar{t}))+\langle\xi(t), y(t)-y(\bar{t})\rangle+\nu(\delta) \mu(\mathcal{S} \cap[\bar{t}, t \wedge \kappa]) \\
& \leq \int_{\mathcal{S} \cap[\bar{t}, t]}\langle\xi(t), w(s)\rangle d s \\
& +\int_{[\bar{t}, t] \backslash \mathcal{S}}\langle\xi(t), \dot{\hat{x}}(s)\rangle d s+\nu(\delta) \mu(\mathcal{S} \cap[\bar{t}, t \wedge \kappa]) .
\end{aligned}
$$

As in the proof of [16, Theorem 5], it follows that $\xi(t)$ is a convex combination of $m$ vectors $\xi_{\alpha} \in N_{K}^{1}\left(y^{\alpha}\right)$, with $y^{\alpha} \in \pi_{\partial K}(z(t))$, such that for all $s \in[\bar{t}, \kappa]$ and $\alpha \in\{1, \cdots, m\}$ we have $\left\|y^{\alpha}-\hat{x}(s)\right\| \leq \eta$, where $1 \leq m \leq n+1$. Then, from (37) together with (34), we obtain that

$$
\begin{aligned}
& \tilde{d}_{K}(x(t)) \leq \sum_{\alpha=1}^{m} \lambda_{\alpha} \int_{\mathcal{S} \cap[\bar{t}, t]}\left\langle\xi_{\alpha}, w(s)\right\rangle d s \\
& +\sum_{\alpha=1}^{m} \lambda_{\alpha} \int_{[\bar{t}, t] \backslash \mathcal{S}}\left\langle\xi_{\alpha}, \dot{\hat{x}}(s)\right\rangle d s+\nu(\delta) \mu(\mathcal{S} \cap[\bar{t}, t \wedge \kappa]) \\
& \leq(\nu(\delta)-\rho) \mu(\mathcal{S} \cap[\bar{t}, t])<0 .
\end{aligned}
$$

Case 2. $t \in(\kappa, \bar{\delta}]$ : By the mean-value theorem, for some $z(t) \in[\hat{x}(t), y(t)]$ and $\xi(t) \in \partial \tilde{d}_{K}(z(t))$,

$$
\tilde{d}_{K}(y(t))=\tilde{d}_{K}(\hat{x}(t))+\langle\xi(t), y(t)-\hat{x}(t)\rangle .
$$

Then,

$$
\begin{aligned}
& \tilde{d}_{K}(x(t)) \leq \tilde{d}_{K}(y(t))+\nu(\delta) \mu(\mathcal{S} \cap[\bar{t}, t \wedge \kappa]) \\
& =\tilde{d}_{K}(\hat{x}(t))+\langle\xi(t), y(t)-\hat{x}(t)\rangle \\
& +\nu(\delta) \mu(\mathcal{S} \cap[\bar{t}, t \wedge \kappa]) \\
& =\tilde{d}_{K}(\hat{x}(t))+\int_{\bar{t}}^{t}\langle\xi(t), \dot{y}(s)-\dot{\hat{x}}(s)\rangle d s \\
& +\nu(\delta) \mu(\mathcal{S} \cap[\bar{t}, t \wedge \kappa]) \\
& =\tilde{d}_{K}(\hat{x}(t))+\int_{\mathcal{S} \cap[\bar{t}, t]}\langle\xi(t), w(s)-\dot{\hat{x}}(s)\rangle d s \\
& +\nu(\delta) \mu(\mathcal{S} \cap[\bar{t}, t \wedge \kappa])
\end{aligned}
$$

As in the first case, $\xi(t)$ is a convex combination of $m$ vectors $\xi_{\alpha} \in N_{K}^{1}\left(y^{\alpha}\right)$, with $y^{\alpha} \in \pi_{\partial K}(z(t))$, such that for all $s \in[\bar{t}, \kappa]$ and $\alpha \in\{1, \cdots, m\}$ we have $\left\|y^{\alpha}-\hat{x}(s)\right\| \leq \eta$, where $1 \leq m \leq n+1$. Then, from (38), we obtain that

$$
\begin{aligned}
& \tilde{d}_{K}(x(t)) \leq \tilde{d}_{K}(\hat{x}(t))+\nu(\delta) \mu(\mathcal{S} \cap[\bar{t}, t \wedge \kappa]) \\
& +\sum_{\alpha=1}^{m} \lambda_{\alpha} \int_{\mathcal{S} \cap[\bar{t}, t]}\left\langle\xi_{\alpha}, w(s)-\dot{\hat{x}}(s)\right\rangle d s \\
& \leq \tilde{d}_{K}(\hat{x}(t))-\rho \mu(\mathcal{S} \cap[\bar{t}, \kappa])+\nu(\delta) \mu(\mathcal{S} \cap[\bar{t}, t \wedge \kappa]) \\
& \leq \tilde{d}_{K}(\hat{x}(t))-(\rho-\nu(\delta))\left[\Gamma \max _{s \in[\bar{t}, T]} d_{K}(\hat{x}(s))+\varepsilon^{\prime}\right] \\
& <0 .
\end{aligned}
$$

This completes the proof.

Proof of Theorem 3. Let $r:=\left(1+\lambda_{0} \tau+r_{0}\right) e^{\sigma T}$ and $\delta=\delta(r), c=c(r)>0$ be as in Lemma 6. Fix $t_{0} \in[0, T]$ and an $F$-trajectory $\hat{x}(\cdot)$ such that $\hat{x}_{t_{0}}$ is $\lambda_{0}$-Lipschitz and $\hat{x}\left(t_{0}\right) \in K \cap r_{0} B$. Let $N$ be the smallest integer satisfying $\left(t_{0}+N \delta\right) \wedge T=T$. Set $t_{i}=\left(t_{0}+i \delta\right) \wedge T$ for all $i=1, \cdots N$. Fix any $\varepsilon_{0}>0$. Lemma 6 assures that, for any sequence of positive numbers $\varepsilon_{1}, \cdots, \varepsilon_{N-1}$, there exists a sequence of $F$-trajectories $\left\{x_{0}(\cdot)=\hat{x}(\cdot), x_{i}(\cdot)\right.$ : $i=1, \cdots, N\}$ such that for all $i=1, \cdots, N$

$$
\left\{\begin{array}{l}
x_{i}(t)=x_{i-1}(t), \quad \forall t \in\left[t_{0}-\tau, t_{i-1}\right] \\
x(t) \in \operatorname{Int} K, \quad \forall t \in\left(t_{0}, t_{i}\right] \\
\left\|x_{i, t}-x_{i-1, t}\right\|_{C} \leq c \max _{t \in\left[t_{0}, T\right]} d_{K}\left(x_{i-1}(t)\right)+\varepsilon_{i-1} .
\end{array}\right.
$$

Lemma 6 is applied recursively on the interval $\left[\left(t_{i-1}-\tau\right) \wedge T, T\right]$ with reference trajectory $x_{i-1}(\cdot)$ restricted to this interval, for $i=1, \cdots, N$. Note that, at each stage of this recursive construction, the same constant $\delta$ and $c$ are used; this is justified by the fact that $x_{i-1, t_{i-1}} \in r B_{C}$, for all $i=1, \cdots, N$. Call $x(\cdot)=x_{N}(\cdot)$, then $x_{t_{0}}=\hat{x}_{t_{0}}$ and $x(t) \in \operatorname{Int} K$ for every $t \in\left(t_{0}, T\right]$. Using the same arguments as in [16, Theorem 5], we prove the existence of some $C>0$, independent from $t_{0}, \hat{x}(\cdot)$ and $\varepsilon_{0}$ such that

$$
\left\|x_{t}-\hat{x}_{t}\right\|_{C} \leq C\left(\max _{t \in\left[t_{0}, T\right]} d_{K}(\hat{x}(t))+\varepsilon_{0}\right) .
$$

This completes the proof.

\section{E. Proof of Corollary 1.}

Fix a relaxed feasible $F$-trajectory $\bar{x}(\cdot)$ such that $\bar{x}_{t_{0}}$ is $\lambda_{0}$-Lipschitz and $\bar{x}\left(t_{0}\right) \in K \cap r_{0} B$, and $\delta>0$. Let $C$ be as in Theorem 3. By Theorem 2, there exists an $F$-trajectory $\hat{x}(\cdot)$ on $\left[t_{0}-\tau, T\right]$ satisfying $\hat{x}_{t_{0}}=\bar{x}_{t_{0}}$ and $\left\|\hat{x}_{t}-\bar{x}_{t}\right\|_{C} \leq \delta / 3 C$ for all $t \in\left[t_{0}, T\right]$. By Theorem 3, for every $\varepsilon_{0}>0$, there exists a feasible $F$ trajectory $x(\cdot)$ such that $x_{t_{0}}=\hat{x}_{t_{0}}, x\left(\left(t_{0}, T\right]\right) \in \operatorname{Int} K$ 
and $\left\|x_{t}-\hat{x}_{t}\right\|_{C}<C\left(\max _{t \in\left[t_{0}, T\right]} d_{K}(\hat{x}(t))+\varepsilon_{0}\right)$. Remark that $d_{K}(\hat{x}(t)) \leq d_{K}(\bar{x}(t))+\left\|\hat{x}_{t}-\bar{x}_{t}\right\|_{C}<\delta / 3 C$. Set $\varepsilon_{0}=\delta / 3 C$. Then, $\left\|x_{t}-\bar{x}_{t}\right\|_{C} \leq \delta$ for all $t \in\left[t_{0}, T\right]$.

\section{F. Proof of Lemma 2.}

Lemma 7: Let $\lambda>0$ and assume (H1)-(H4) and $\left(I P_{\text {rel }}^{\lambda}\right)$. Then for every $R>0, \bar{\psi} \in \mathcal{K}_{\lambda} \cap R B_{C}$ and every $\bar{t} \in[0, T]$ there exists $\rho_{\bar{t}, \bar{\psi}}>0$ such that $\forall v \in \operatorname{co} F(\bar{t}, \bar{\psi})$ with $\max _{n \in N_{K}^{1}(\bar{\psi}(0))}\langle n, v\rangle \geq 0$ we can find $w \in \operatorname{Liminf}_{(s, \phi) \rightarrow(\bar{t}, \bar{\psi})} \operatorname{co} F(s, \phi)$ satisfying

$$
\max \left\{\langle n, w\rangle,\langle n, w-v\rangle \mid n \in N_{K}^{1}(\bar{\psi}(0))\right\} \leq-2 \rho_{\bar{t}, \bar{\psi}} .
$$

Proof. The proof follows the same lines as [17, Proof of Lemma 3.7].

Proof of Lemma 2. Let $R>0, \bar{t} \in[0, T]$ and $\bar{\psi} \in \mathcal{K}_{\lambda} \cap R B_{C}$ be fixed and let $\rho_{\bar{t}, \bar{\psi}}$ be as in Lemma 7 . We claim the existence of $\eta_{\bar{t}, \bar{\psi}}>0$ such that for every $t \in B\left(\bar{t}, \eta_{\bar{t}, \bar{\psi}}\right)$, every $\psi \in \mathcal{K}_{\lambda} \cap B_{C}\left(\bar{\psi}, \eta_{\bar{t}, \bar{\psi}}\right)+\eta_{\bar{t}, \bar{\psi}} B_{C}$ and every $v \in \operatorname{co} F(t, \psi)$ with

$$
\max _{n \in N_{K}^{1}(x), x \in \partial K \cap B\left(\psi(0), \eta_{\bar{t}, \bar{\psi}}\right)}\langle n, v\rangle \geq 0,
$$

there exists $w \in \operatorname{co} F(t, \psi)$ satisfying

$$
\left\{\begin{array}{l}
\langle n, w\rangle \leq-\rho_{\bar{t}, \bar{\psi}} \text { and }\langle n, w-v\rangle \leq-\rho_{\bar{t}, \bar{\psi}} \\
\forall n \in N_{K}^{1}(x), \forall x \in \partial K \cap B\left(\psi(0), \eta_{\bar{t}, \bar{\psi}}\right) .
\end{array}\right.
$$

Suppose by contradiction that there exist $t_{i} \rightarrow \bar{t}, \psi_{i} \rightarrow \bar{\psi}, \quad v_{i} \in \operatorname{co} F\left(t_{i}, \psi_{i}\right), x_{i} \rightarrow \partial K \quad \bar{\psi}(0)$ and $n_{i} \in N_{K}^{1}\left(x_{i}\right)$ such that $\left\langle n_{i}, v_{i}\right\rangle \geq 0$ and for every $w_{i} \in \operatorname{co} F\left(t, \psi_{i}\right)$ we can find $x_{i}^{\prime} \rightarrow_{\partial K} \bar{\psi}(0), n_{i}^{\prime} \in N_{K}^{1}\left(x_{i}^{\prime}\right)$ satisfying

$$
\left\langle n_{i}^{\prime}, w_{i}\right\rangle \vee\left\langle n_{i}^{\prime}, w_{i}-v_{i}\right\rangle>-\rho_{\bar{t}, \bar{\psi}}
$$

Since $F$ is upper semicontinuous at every point of $[0, T] \times \mathcal{K}_{\lambda}$, so is co $F$. taking subsequences and keeping the same notations we may assume that $v_{i}$ converge to some $v \in \operatorname{co} F(\bar{t}, \bar{\psi}), n_{i} \rightarrow n$ and $n_{i}^{\prime} \rightarrow n^{\prime}$. Since the map $x \rightsquigarrow N_{K}^{1}(x)$ is upper semicontinuous, we have $n, n^{\prime} \in N_{K}^{1}(\bar{\psi}(0))$ and $\langle n, v\rangle \geq 0$. Then $\max _{n \in N_{K}^{1}(\bar{\psi}(0))}\langle n, v\rangle \geq 0$. Consider $w$ as in (39) corresponding to this $v$ and let $w_{i} \in \operatorname{co} F\left(t, \psi_{i}\right)$ be such that $w_{i} \rightarrow w$. From (40) we deduce that $\left\langle n^{\prime}, w\right\rangle \vee\left\langle n^{\prime}, w-v\right\rangle \geq-\rho_{\bar{t}, \bar{\psi}}$, contradicting the choice of $w$. The rest of the proof is similar to Step 3 of Lemma 1.

\section{G. Proof of Theorem 5.}

The proof of Theorem 5 is a straightforward consequence of Theorem 4. Indeed, fix $t_{0} \in[0, T]$ and let us introduce for every $(t, \psi) \in\left[t_{0}, T\right] \times$ $\mathcal{C}\left([-\tau, 0], \mathbb{R}^{n}\right)$ the set-valued map $F$ defined by $F(t, \psi):=\mathcal{F}(t, \psi(0), \psi(-\tau))$. It is easy to see that under the assumptions (A1)-(A4), the set-valued map $F$ verifies (H1)-(H4). In addition, we can show that, under $\left(I P_{e q}^{\lambda}\right)$, condition $\left(I P_{\text {rel }}^{\lambda}\right)$ holds true. In fact, let $\psi \in \mathcal{K}_{\lambda}$. By definition of $\mathcal{K}_{\lambda}$, we have $\psi(0) \in \partial K$ and $\psi(-\tau) \in \psi(0)+\lambda \tau B$. Then, from $\left(I P_{e q}^{\lambda}\right)$, for every $v \in F(t, \psi) \equiv$ $\mathcal{F}(t, \psi(0), \psi(-\tau))$ such that $\max _{n \in N_{K}^{1}(\psi(0))}\langle n, v\rangle \geq 0$ there exists $w \in \operatorname{Liminf}_{(s, z, \xi) \rightarrow(t, \psi(0), \psi(-\tau))} \operatorname{co} \mathcal{F}(s, z, \xi)$ satisfying

$$
\max _{n \in N_{K}^{1}(\psi(0))}\langle n, w-v\rangle<0 .
$$

From the inclusion

$$
\begin{gathered}
\operatorname{Liminf}_{(s, z, \xi) \rightarrow(t, \psi(0), \psi(-\tau))} \operatorname{co} \mathcal{F}(s, z, \xi) \subset \\
\operatorname{Liminf}_{(s, \phi) \rightarrow(t, \psi)} \operatorname{co} F(s, \phi),
\end{gathered}
$$

we deduce that $\left(I P_{r e l}^{\lambda}\right)$ holds true. Hence, Theorem 4 concludes the proof.

\section{REFERENCES}

[1] W. G. Aiello and H. I. Freedman. A time-delay model of singlespecies growth with stage structure. Mathematical Biosciences, 101:139-153, 1990

[2] J. P. Aubin. Viability theory. Birkhäuser, Basel, 1991.

[3] J. P. Aubin and H. Frankowska. Set-Valued Analysis. Birkhaüser, Boston, 1990.

[4] J.P. Aubin and P. Saint-Pierre. An introduction to viability theory and management of renewable resources. In J. Kropp and J. Scheffran, editors, Advanced Methods for Decision Making and Risk Management, pages 43-80. Nova Science Publishers, 2007.

[5] R. J. Aumann. Integrals of set-valued functions. J. Mathematical Analysis and Applications, 12:1-12, 1965.

[6] N. Bekiaris-Liberis and M. Krstic. Nonlinear Control Under Nonconstant Delays. Society for Industrial and Applied Mathematics, Philadelphia, PA, 2013.

[7] C. Bernard and S. Martin. Building strategies to ensure language coexistence in presence of bilingualism. Applied Mathematics and Computation, 218(17):8825-8841, 2012.

[8] P. Bettiol, A. Bressan, and R. B. Vinter. On trajectories satisfying a state constraint: $W^{1,1}$ estimates and counter-examples. SIAM J. Control Optimal, 48:4664-4679, 2010.

[9] P. Bettiol, H. Frankowska, and R. B. Vinter. $L^{\infty}$ estimates on trajectories confined to a closed subset. J. Differential Equations, 252:1912-1933, 2012.

[10] P. Bettiol, H. Frankowska, and R.-B. Vinter. Improved sensitivity relations in state constrained optimal control. Applied Mathematics \& Optimization, 71(2):353-377, 2015.

[11] F. H. Clarke. Optimization and nonsmooth analysis. SIAM, Philadelphia, 1990.

[12] A. F. Filippov. Classical solutions of differential equations with multivalued right hand side. SIAM J. Control Optimal, 5:609621, 1967. 
[13] F. Forcellini and F. Rampazzo. On nonconvex differential inclusions whose state is constrained in the closure of an open set. applications to dynamic programming. J. Differential and Integral Equations, 12(4):471-497, 1999.

[14] H. Frankowska. A priori estimates for operational differential inclusions. J. Differential Equations, 84:100-128, 1990.

[15] H. Frankowska, E. M. Marchini, and M. Mazzola. A relaxation result for state constrained inclusions in infinite dimension. Mathematical Control and Related Fields, 6(1):113-141, 2016.

[16] H. Frankowska and M. Mazzola. Discontinuous solutions of Hamilton-Jacobi-Bellman equation under state constraints. Calculus of Variations and Partial Differential Equations, 46:725747, 2013.

[17] H. Frankowska and M. Mazzola. On relations of the adjoint state to the value function for optimal control problems with state constraints. Nonlinear Differential Equations and Applications, 20:361-383, 2013.

[18] H. Frankowska and M. Quincampoix. Viability kernels of differential inclusions with constraints: algorithm and applications. $J$. Math. Systems, Estimation and Control, 1:371-388, 1991.

[19] H. Frankowska and F. Rampazzo. Filippov's and FilippovWazewski's theorems on closed domains. J. Differential Equations, 161:449-478, 2000 .

[20] H. Frankowska and R. B. Vinter. Existence of neighboring feasible trajectories: Applications to dynamic programming for state-constrained optimal control problems. J. Optimization Theory and Applications, 104(1):21-40, 2000.

[21] G. Haddad. Monotone viable trajectories for functional differential inclusions. J. Differential Equations, 42:1-24, 1981.

[22] I. Haidar, I. Alvarez, and A.C. Prévot. Mathematical modeling of an urban pigeon population subject to local management strategies. Mathematical Biosciences, 288:71 - 83, 2017.

[23] J.K. Hale and S.M. Verduyn Lunel. Introduction to functional differential equations, volume 99. Springer-Verlag, 1993.

[24] Y. Kuang. Delay differential equations with application in population dynamics. Academic Press, Boston, 1993.

[25] M. De Lara and V. Martinet. Multi-criteria dynamic decision under uncertainty: A stochastic viability analysis and an application to sustainable fishery management. Mathematical Biosciences, 217(2): 118 - 124, 2009.

[26] H.M. Regan, Y. Ben-Haim, B. Langford, W. Wilson, and P. Lundgerg. Robust decision-making under severe uncertainty for conservation management. Ecological Applications, 15(4):1471-1477, 2005.

[27] J.-B. Rouquier, I. Alvarez, R. Reuillon, and P.-H. Wuillemin. A kd-tree algorithm to discover the boundary of a black box hypervolume. Annals of Mathematics and Artificial Intelligence, 75(3):335-350, 2015.

[28] P. Saint-Pierre. Approximation of the viability kernel. Applied Mathematics \& Optimisation, 29(2):187-209, 1994.

[29] M. Sicard, N. Perrot, R. Reuillon, S. Mesmoudi, I. Alvarez, and S. Martin. A viability approach to control food processes: Application to a camembert cheese ripening process. Food Control, 23(2):312 - 319, 2012.

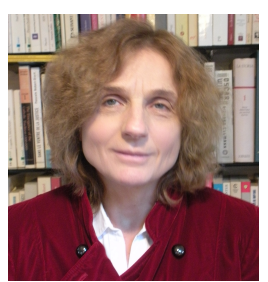

Hélène Frankowska has completed her habilitation (doctorat ès sciences) of Mathematics in 1984 at University of Paris Dauphine, France. She is currently Directeur de Recherche at CNRS and University of Paris 6. She has held visiting positions at Centre de Recherche Mathématiques, University of Montréal (Canada), International Institute for Applied Systems Analysis (Austria); Istituto Nazionale di Alta Matematica "F. Severi”, Roma (Italy); Scuola Normale Superiore di Pisa (Italy); Scuola Internazionale Superiore di Studi Avanzati, Trieste (Italy); Università di Roma Tor Vergata (Italy). She was invited sectional speaker at International Congress of Mathematicians ICM 2010 in Hyderabad. Her research activities are focused on optimal control, both in the deterministic and stochastic framework, control of systems under state constraints, viability theory, differential inclusions, setvalued and variational analysis. On these topics, she has published more than 170 research papers in international journals, books and conferences proceedings. She is also a co-author of the book "Set-Valued Analysis", published in two editions in 1990 and 2009 (Modern Birkhaüser Classics). She is currently serving as Editor in several peer reviewed journals, in particular, as Corresponding Editor of SIAM Journal on Control and Optimization (SICON), Division Editor of Journal of Mathematical Analysis and Applications (JMAA), Associate Editor of Mathematical Control and Related Fields (MCRF). She is a Board member of the Academic Press Mathematics in Science and Engineering Book Series.

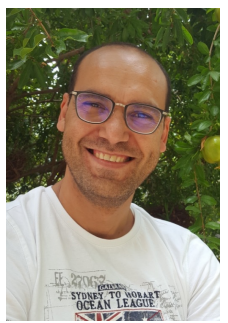

Ihab Haidar was born in Beirut, Lebanon, in 1983. He received the Master's degree in mathematics from the University of Aix-Marseille 1, France, in 2008 and the Ph.D. degree from the University of Montpellier 2, France, in 2011. Since then he has been post-doc in different places (Laboratoire des Signaux et Systèmes-CentraleSupélec, Institut de Mathématiques de Jussieu-Paris 6, Laboratoire Quartz-ENSEA). He is currently Maître De Conférences at ENSEA. His main research interests include Nonlinear control theory, Time delay systems and Systems biology. 